\section{The infectious diseases specialty in South Africa is in crisis}

To the Editor: HIV, tuberculosis, lower respiratory tract infections and diarrhoeal diseases are among the leading causes of death in South Africa (SA), ${ }^{[1]}$ and account for a large proportion of healthcare expenditure. A large and expanding national antiretroviral treatment programme has been associated with a decline in the incidence of $\mathrm{HIV}^{[2]}$ and tuberculosis, ${ }^{[3]}$ as well as declining HIV and tuberculosis-related mortality. ${ }^{[1]}$ However, complex problems related to the heavy burden of these and other infectious diseases (IDs), which require input and co-ordination from skilled specialists, persist in the healthcare system and cannot always be addressed by generic programme-level guidance. It is therefore vital that SA has the appropriate number of specialists to cope with this enormous burden of disease.

\section{The role of ID specialists}

ID specialists are trained to perform multiple tasks in the healthcare system. They are mainly employed at tertiary hospitals, where a key role is to solve complex infection-related clinical problems. However, such is the burden of infection in SA's hospitals that a strong case has been made for employing ID specialists in secondary-level hospitals too. As infections are very common in hospitalised patients, ID specialists routinely provide in-reach services in their own hospital, e.g. ID consultations and antimicrobial stewardship (AMS) ward rounds on surgical, obstetric and intensive care wards, and outreach at lower-level hospitals and even at private hospitals that do not have ID specialists.

ID specialists are often called upon to give expert advice to committees, ranging from infection prevention and control (IPC) and AMS committees in hospitals, to provincial and national committees making important policy decisions in areas such as essential medicines, HIV, tuberculosis, antibiotic resistance, vaccines, and emerging and re-emerging infections such as Crimean-Congo fever and other viral haemorrhagic fevers. ID specialists are responsible for training the next generation of doctors in the management of infection, specialists and non-specialists alike, as well as other cadres of staff including nurses and pharmacists.

There is overwhelming evidence that ID specialists add value to the healthcare system. Specifically, ID consultations are associated with shorter hospital stays, lower antimicrobial prescribing, lower readmission rates, decreased mortality and lower healthcare costs. ${ }^{[4-8]}$ A specific example of cost saving is the ZAR2.8 million saved on antibiotic costs alone over 4 years on just four wards by the multidisciplinary AMS programme led by ID specialists at Groote Schuur Hospital in Cape Town. ${ }^{[9]}$

As a stopgap while the shortfall of ID expertise countrywide is addressed, ID specialists have taken the innovative step of providing a nationwide service, via a dedicated WhatsApp group, to deliver timely infection-related advice to clinicians who lack access to specialists. Advice is delivered by 14 ID specialists, usually within 15 minutes, and the whole system runs on goodwill and therefore at zero cost to the government or donors. While such developments are welcome, they are merely a temporary measure until such time as adequate numbers of specialists are trained and employed.

SA is home to some world-renowned ID research institutes. There is no validated metric for the contribution of ID specialists to research in SA. However, a recent PubMed search for studies in humans with the MESH term 'South Africa' for the year 2017 produced 1650 articles. Refining the search to articles including the terms 'infection' or 'tuberculosis' or 'HIV' or 'antibiotics' yielded 660 articles, suggesting that a large proportion of SA's scientific research output is in some way related to infectious diseases. Furthermore, clinical ID specialists join their university counterparts in leading many of the major research groups that drive this critical research enterprise.

\section{The ID specialty in SA}

ID was recognised as a subspecialty of Medicine and Paediatrics in 2005. Fellows of the colleges of Physicians and Paediatricians (FCP (SA) or FC Paeds (SA)) are required to enter a 2- to 3-year training programme towards the final exit exam to obtain the Cert ID (SA) qualification. More rarely, fellows of the College of Pathologists (SA) in Microbiology qualify to sit the Cert ID (SA) examination after 18 months of clinical ID training. Paradoxically, despite ID contributing so massively to SA's total burden of disease, ${ }^{[10]}$ the ID subspecialty was developed at a late stage. Lack of leadership, and few specialists to direct proper management of infection, may have been detrimental to the SA public health system, resulting in deaths and contributing to the development of multidrug-resistant

Table 1. Breakdown of subspecialists registered with the Health Professions Council of South Africa*

\begin{tabular}{|c|c|c|c|c|c|c|c|c|c|c|c|}
\hline Specialty & Subspecialty & $\mathrm{EC}$ & FS & G & KZN & $\mathbf{L}$ & MP & NW & $\mathrm{NC}$ & WC & Total \\
\hline \multirow[t]{7}{*}{ Adult medicine } & Cardiology & 13 & 11 & 109 & 38 & 1 & 2 & 2 & 1 & 61 & 238 \\
\hline & Endocrinology & 2 & 4 & 28 & 15 & 0 & 0 & 0 & 0 & 13 & 62 \\
\hline & Gastroenterology & 4 & 1 & 51 & 16 & 0 & 0 & 1 & 0 & 25 & 98 \\
\hline & Infectious diseases & 2 & 2 & 13 & 7 & 0 & 0 & 0 & 0 & 17 & 41 \\
\hline & Nephrology & 4 & 6 & 52 & 27 & 0 & 0 & 1 & 1 & 29 & 120 \\
\hline & Pulmonology & 5 & 3 & 54 & 12 & 0 & 0 & 0 & 0 & 36 & 110 \\
\hline & Rheumatology & 3 & 4 & 29 & 14 & 0 & 0 & 0 & 0 & 26 & 76 \\
\hline \multirow[t]{7}{*}{ Paediatrics } & Cardiology & 4 & 3 & 19 & 4 & 0 & 2 & 1 & 1 & 9 & 43 \\
\hline & Endocrinology & 0 & 0 & 8 & 4 & 0 & 0 & 0 & 0 & 8 & 20 \\
\hline & Gastroenterology & 0 & 2 & 11 & 1 & 0 & 0 & 0 & 0 & 5 & 19 \\
\hline & Infectious diseases & 2 & 0 & 5 & 4 & 0 & 0 & 0 & 0 & 12 & 23 \\
\hline & Nephrology & 2 & 1 & 10 & 3 & 0 & 0 & 0 & 0 & 8 & 24 \\
\hline & Pulmonology & 0 & 0 & 23 & 6 & 4 & 0 & 0 & 0 & 36 & 69 \\
\hline & Rheumatology & 0 & 0 & 4 & 1 & 0 & 0 & 0 & 0 & 4 & 9 \\
\hline
\end{tabular}


infections. ${ }^{[11,12]}$ Despite this, there are fewer ID subspecialists today than in any other of the major medical specialties (Table 1), and those who do qualify as ID specialists are often forced into private practice owing to the lack of ID posts in the public sector, and the lack of understanding of the importance of having ID-trained specialists at our secondary-level institutions. In effect, we are now training ID subspecialists with public money for the private sector at no cost to the latter, and not recognising the desperate need for trained ID specialists in the public service.

\section{What needs to be done?}

This letter has emphasised the extensive value that ID specialists add to the healthcare service. We call upon the Minister of Health to engage with ID leadership in SA to address its shortage of ID specialists, with particular emphasis on ensuring equity countrywide. Four of our nine provinces have no ID specialist employed. Moreover, the healthcare system's growing needs in the realms of IPC, AMS, transitions of care, population health, and most importantly clinical outcomes, justify employing young ID specialists in both urban and rural healthcare settings, and not just at tertiary hospitals. We believe that this will significantly reduce morbidity, mortality and healthcare costs, as it has done worldwide.

Undergraduate and postgraduate training in infection management must be strengthened. The work currently being undertaken by the Ministerial Advisory Committee on Antimicrobial Resistance to strengthen curricula towards achieving appropriate antibiotic use needs to be expanded to the wider field of infectious diseases and their prevention and treatment.

Professional groups such as the Federation of Infectious Diseases Societies of Southern Africa and its affiliated societies stand ready to partner with the national government to expand mentorship and scholarship activities.

We call upon universities and other funders to support an expanded programme of ID-related research, which has hitherto focused almost entirely on HIV, tuberculosis, and malaria. SA's massive burden of community-acquired (including neglected tropical diseases such as schistosomiasis) and hospital-acquired infection must be recognised. Government and research funders need to expand their portfolios to address these burdens too.

\section{Conclusions}

The specialty of ID is in crisis in SA. Despite the enormous burden of infectious disease, there are fewer trained specialists than in any other major discipline, and a shortage of training posts means that this is unlikely to reverse in the near future. It is vital that the National Department of Health recognises this, and the crucial role that ID specialists play, first and foremost in caring for patients, training cadres of staff, directing infection-related research, and shaping important health policy decisions for the country and its provinces. This must be coupled with immediate attention to providing an increase in specialist training posts, and positions in the public sector for those completing training. The number of ID specialists must mirror the burden of infectious diseases in SA, so as to deliver the service provision SA needs.

A full list of signatories to this letter is available (http://www.samj. org.za/public/sup/signatories_to_id_opinion_piece.pdf).

\section{Tom Boyles}

President, Infectious Diseases Society of Southern Africa (IDSSA)

tomboyles@yahoo.com

\section{Marc Mendelson}

President, International Society for Infectious Diseases (ISID)

\section{Nelesh Govender}

President, Federation of Infectious Diseases Societies of Southern Africa (FIDSSA)

\section{Nicolette du Plessis}

President, Southern African Society for Paediatric Infectious Diseases (SASPID)

1. Pillay-van Wyk V, Msemburi W, Laubscher R, et al. Mortality trends and differentials in South Africa from 1997 to 2012: Second National Burden of Disease Study. Lancet Glob Health 2016;4(9):e642-e653. https://doi.org/10.1016/s2214-109x(16)30113-9

2. Human Sciences Research Council. 5th National HIV Prevalence Survey. 2017. http://www.hsrc.ac.za/ uploads/pageContent/9234/SABSSMV_Impact_Assessment_Summary_ZA_ADS_cleared_PDFA4. pdf (accessed 8 August 2019).

3. Nanoo A, Izu A, Ismail NA, et al. Nationwide and regional incidence of microbiologically confirmed pulmonary tuberculosis in South Africa, 2004 - 12: A time series analysis. Lancet Infect Dis 2015;15(9):1066-1076. https://doi.org/10.1016/s1473-3099(15)00147-4

4. Schmitt S, MacIntyre AT, Bleasdale SC, et al. Early infectious diseases specialty intervention is associated with shorter hospital stays and lower readmission rates: A retrospective cohort study. Clin Infect Dis 2019;68(2):239-246. https://doi.org/10.1093/cid/ciy494

5. Schmitt S, McQuillen DP, Nahass R, et al. Infectious diseases specialty intervention is associated Schmitt S, McQuillen DP, Nahass R, et al. Infectious diseases specialty intervention is associated
with decreased mortality and lower healthcare costs. Clin Infect Dis 2014;58(1):22-28. https://doi. with decreased mortality and lower healthcare costs. Clin Infect Dis 2014;58(1):22-28. https://doi. org/10.1093/cid/cit610

6. Hamandi B, Husain S, Humar A, Papadimitropoulos EA. Impact of infectious disease consultation on the clinical and economic outcomes of solid organ transplant recipients admitted for infectious complications. Clin Infect Dis 2014;59(8):1074-1082. https://doi.org/10.1093/cid/ciu522

7. Butt AA, Al Kaabi N, Saifuddin M, et al. Impact of infectious diseases team consultation on antimicrobial use, length of stay and mortality. Am J Med Sci 2015;350(3):191-194. https://doi. org/10.1097/maj.0000000000000546

8. Rieg S, Kupper MF. [Impact of an infectious diseases consultation service on the quality of care and the survival of patients with infectious diseases]. Z Evid Fortbild Qual Gesundhwes 2015;109(7):500-510. https://doi.org/10.1016/j.zefq.2015.09.008

9. Boyles TH, Naicker V, Rawoot N, Raubenheimer PJ, Eick B, Mendelson M. Sustained reduction in antibiotic consumption in a South African public sector hospital; Four year outcomes from the Groote Schuur Hospital antibiotic stewardship program. S Afr Med J 2017;107(2):115-118. https://doi. Groote Schur Hospital antibiotic stew

10. Mayosi BM, Lawn JE, van Niekerk A, Bradshaw D, Abdool Karim SS, Coovadia HM. Health in South Africa: Changes and challenges since 2009. Lancet 2012;380(9858):2029-2043. https://doi.org/10.1016/ s0140-6736(12)61814-5

11. Mehta U, Durrheim DN, Blumberg L, et al. Malaria deaths as sentinel events to monitor healthcare delivery and antimalarial drug safety. Trop Med Int Health 2007;12(5):617-628. https://doi. org/10.1111/j.1365-3156.2007.01823.x

12. Paruk F, Richards G, Scribante J, Bhagwanjee S, Mer M, Perrie H. Antibiotic prescription practices and their relationship to outcome in South Africa: Findings of the prevalence of infection in South African intensive care units (PISA) study. S Afr Med J 2012;102(7):613-616.

S Afr Med J 2019;109(9):620-621. https://doi.org/10.7196/SAMJ.2019.v109i9.14297 